\title{
Política cultural y género, un acercamiento a partir de dos casos en América Latina
}

\author{
Diana Bento de Mello y Alejandra Vázquez² \\ ${ }^{1}$ Instituto de Ciencias Antropológicas, Facultad de Filosofía y Letras, Universidad de Buenos Aires. Buenos \\ Aires, Argentina. \\ Correo electrónico: didibmello@gmail.com \\ 2 Instituto de Ciencias Antropológicas, Facultad de Filosofía y Letras, Universidad de Buenos Aires. Buenos \\ Aires, Argentina. \\ Correo electrónico: ale.vazquez.cepeda@gmail.com
}

Recibido:

noviembre de 2019

Aceptado:

abril de 2020

doi: $10.34096 /$ runa.v41i2.8852

\section{Resumen}

El artículo analiza cómo se expresa la cuestión de género en el quehacer cotidiano y en la gestión de políticas de la cultura orientadas al lenguaje expresivo de la música en Argentina y en Brasil. Mediante el enfoque antropológico se analizan datos construidos a partir de dos casos: el Programa Nacional de Coros y Orquestas Infantiles y Juveniles del Bicentenario en Buenos Aires y el samba en tanto movimiento cultural contemporáneo de artistas, trabajadorxs de la cultura y gestores culturales de Río de Janeiro. Concibiendo las políticas públicas y culturales como un campo de disputa y articulación entre la sociedad civil y el Estado, y pensando la dimensión de género como constitutiva de las relaciones sociales, postulamos que la ausencia de una problematización respecto de las cuestiones de género tiende a reproducir las desigualdades preexistentes y los estereotipos hegemónicos de masculinidades, feminidades y otras identidades de género.

\section{Cultural policy and gender, an approach based on two cases in Latin America}

\begin{abstract}
The article analyzes how the gender issue is expressed in everyday life and in the management of cultural policies oriented to music in Argentina and Brazil. With an anthropological approach, we analyze data from two cases: the National Program of Choirs and Children's and Youth Orchestras of the
\end{abstract}

\section{Palabras clave}

Política cultural; Género; Gestión cultural; Coros y Orquestas Infantiles y Juveniles; Samba

\section{Key words}

Cultural policy; Gender; Cultural Management; Children's and Youth Choirs and Orchestras; Samba 
Bicentennial in Buenos Aires and the Samba as a contemporary cultural movement of artists, cultural workers and cultural managers of Rio de Janeiro. Conceiving public and cultural policies as a field of dispute and of articulation between civil society and the State, and thinking of the gender dimension as constitutive of social relations, we postulate that the absence of a question regarding gender issues tends to reproduce pre-existing inequalities and hegemonic stereotypes of masculinities, feminities and other gender identities.

\section{Política cultural e gênero, uma aproximação a partir de dois casos na América Latina}

\section{Resumo}

Palavras-chave

Política cultural; Gênero; Gestão cultural; Coral e Orquestras Infantis e Juvenis; Samba
O artigo analisa como a questão de gênero se expressa no cotidiano da gestão de políticas culturais orientadas à linguagem expressiva da música na Argentina e no Brasil. A través de uma abordagem antropológica se analisam dados construídos a partir de dois casos: o Programa Nacional de Corais e Orquestras Infantis e Juvenis do Bicentenário de Buenos Aires e o Samba em tanto movimento cultural contemporâneo de artistas, trabalhadorxs e gestorxs culturais do Rio de Janeiro. A partir de uma compreensão de que as políticas públicas e culturais são um campo de disputa e articulação entre a sociedade civil e o Estado, e pensando na dimensão de gênero como constitutiva das relações sociais, afirmamos que a ausência de uma problematização das questões de gênero tende a reproduzir estereótipos hegemônicos de masculinidades, feminidades e outras identidades de gênero, assim como a aprofundar as desigualdades já existentes.

\section{Introducción}

En septiembre de 2019 participamos del Encuentro y Foro de Cultura (ENFOCA), en el cual diversos colectivos y organizaciones culturales se reunieron para debatir y construir propuestas de política cultural para la ciudad de Buenos Aires. La convocatoria del encuentro apelaba explícitamente a pensar políticas culturales con una "perspectiva transfeminista". Notamos que las orientaciones de los organismos internacionales también caminan en esa dirección: en 2015, la Asamblea General de la ONU adoptó la "Agenda 2030 para el Desarrollo Sostenible", en la que propone los 17 Objetivos del Desarrollo Sostenible, dentro de los cuales se plantea la "Igualdad de Género" como "la base necesaria para conseguir un mundo pacífico, próspero y sostenible". 1 Tres años después, la Comisión de Cultura de la CGLU (Ciudades y Gobiernos Locales Unidos) publicó un documento orientativo sobre cómo trabajar transversalmente dichos objetivos en el campo cultural, y postuló la necesidad de "Integrar una dimensión de género en la política cultural" (CGLU, 2018, p. 13).

Estos dos ejemplos manifiestan la irrupción de la perspectiva de género y de los feminismos ${ }^{2}$ en las agendas de las políticas culturales a partir de los últimos años de este siglo en nuestra región. Contextualmente, esto también se entiende a partir del creciente activismo del movimiento de mujeres, feministas y 
LGTTTBI3 y la -no siempre- consecuente incorporación en la agenda del Estado de temáticas vinculadas a la promoción y protección de derechos de géneros y sexualidades.

El presente artículo está pensado a partir de discusiones teórico-metodológicas llevadas a cabo en el equipo de investigación 4 del que formamos parte como antropólogas investigadoras de la cultura. Los datos utilizados se basan en dos investigaciones referentes a políticas culturales distintas, desarrolladas en emplazamientos sociales disímiles. Una de las pesquisas se ha desarrollado en el ámbito del Programa Nacional de Coros y Orquestas Infantiles y Juveniles del Bicentenario (en adelante, POyCB) en el Gran Buenos Aires, durante los años 2015 y 2016. La otra -con el trabajo de campo aún en curso desde 2018trabaja con el samba en tanto movimiento cultural contemporáneo de artistas, trabajadorxs5 de la cultura y gestorxs culturales en la ciudad de Río de Janeiro (Brasil) y su vinculación con la gestión de políticas culturales. Si bien estos casos difieren en el tipo de política pública cultural -una estatal, y la otra, pública no estatal-, así como el tipo de intervención de la política cultural y lxs actores sociales involucradxs, tienen en común el uso del lenguaje expresivo de la música.

Parafraseando a Howard Becker (2016), comprendemos que el proceso de razonar a partir de casos específicos nos lleva a un mejor entendimiento de procesos sociales más amplios. Por lo tanto, en este estudio, que toma dos casos bastante diferentes entre sí, es posible abordar la intersección entre género y cultura como parte de dinámicas transversales a nuestra región, y orientarnos -tal como sugiere Becker- hacia un conocimiento más concreto del mundo.

De este modo, el objetivo general del presente artículo es analizar cómo se expresa la cuestión de género en el quehacer cotidiano y en la gestión de políticas de la cultura orientadas al lenguaje expresivo de la música, a partir de dos casos, uno en Argentina y otro en Brasil.

\section{Política cultural y género como cuestión en América latina, una breve historización}

A partir de la segunda mitad del siglo XX, los Estados nacionales comenzaron a desarrollar un interés especial respecto de la dimensión cultural, que implica la constitución de jurisdicciones específicas y/o ministerios de Cultura (García Canclini, 1987), en especial a partir de la acción de organismos especializados como la UNESCO. En ese contexto, la incipiente política cultural de los países de nuestra región se restringía a las artes, al espectáculo o a un cultivo simbólico e intelectual como esfera apartada de la economía o de la sociedad, que concibe "la cultura" como una posible puerta de entrada a la modernidad (Nivón Bolán, 2013). También hacia la década de 1960, a la par de la efervescencia de otros movimientos sociales y políticos, la dimensión de género se impuso junto con el movimiento feminista internacional, intensificado con nuevas demandas y reivindicaciones centradas en el cuerpo y en la sexualidad de las mujeres. Remarcamos que, especialmente en los casos de Argentina y Brasil, este periodo se correspondió con golpes de Estado y dictaduras cívico-militares.

Por lo tanto, es recién a partir de la década de 1980, en el contexto latinoamericano de apertura democrática, que se incorporaron como cuestiones problematizadas por la política pública la igualdad de género y la gestión de la cultura más allá de lo patrimonial y museográfico relacionado con la "alta
3. Utilizamos la sigla LGTTTBI en referencia a la comunidad organizada de lesbianas, gays, trans, travestis, transgénero, bisexuales e intersex.

4. Proyecto UBACyT “La continuidad de las políticas públicas designadas en términos de inclusión y participación, rupturas y reelaboraciones en el nuevo contexto político nacional. Aportes desde una mirada antropológica".

5. El uso de la " $x$ " intenta visibilizar las marcas genéricas del lenguaje y trascender el binomio femenino/masculino, fundante del pensamiento heterosexual, la heteronormatividad obligatoria y la homofobia (Wittig, 2006). 
cultura". En este contexto, el movimiento feminista argentino, en conjunto con diversas organizaciones culturales, inauguraron el Primer Encuentro Nacional de Mujeres en 1986. Un año después se creó la primera Secretaría de Estado de la Mujer (Tarducci y Rifkin, 2010). En el caso de Brasil, la participación de las mujeres fue marcada por la Carta das mulheres brasileiras aos constituintes, que incorporaba en la nueva Constitución de 1988 el conjunto de las reivindicaciones del movimiento feminista y de mujeres (Menicucci de Oliveira, 2013). Asimismo, es necesario precisar que este inicio de democratización cultural en un sentido amplio emergió en el marco de un periodo neoliberal en nuestra región.

Desde el inicio del presente siglo, con el avance de los gobiernos progresistas en nuestra región y sus procesos políticos democratizadores, se comenzaron a implementar algunas transformaciones institucionales significativas hacia el acortamiento de la brecha de la desigualdad y la ampliación de derechos.

En lo que atañe al campo de la cultura, las orientaciones de la UNESCO inauguraron lo que Nivón Bolán (2013) denomina como el paradigma de la diversidad. Según este autor, en América Latina, "el debate sobre la diversidad se encarna como un proceso emancipador político, intelectual y cultural" (p. 29), que tuvo su corolario en la realización del Foro Social Mundial como principal fenómeno intelectual contracultural de cuestionamiento al monoculturalismo neoliberal.

Se puede observar cómo el mismo paradigma de ampliación de derechos con foco en la diversidad orientó también las políticas de sexualidad y género. En Argentina se destacaron, entre otras, el Programa Nacional de Salud Sexual y Procreación Responsable (2003), el Programa Nacional de Educación Sexual Integral (2006), la Ley de protección integral para prevenir, sancionar y erradicar la violencia contra las mujeres en los ámbitos en que desarrollen sus relaciones interpersonales (2009), la Ley de Matrimonio igualitario (2010), la Ley de Identidad de Género (2012), la Ley de Prevención y sanción de la trata de personas y asistencia a sus víctimas (2008 y 2012) y la Ley de Parto Humanizado (2015).

Asimismo, en los últimos años se observa un periodo de restauración neoliberal y un retroceso en las políticas orientadas hacia la diversidad y la expansión de derechos. En Brasil, esto se inicia en 2016 con el proceso de impeachement y la posterior destitución de la presidenta electa Dilma Rousseff, y llegó a su ápice en 2018, con el mandato de prisión al expresidente Luiz Inácio Lula da Silva y la posterior victoria electoral de Jair Bolsonaro. En Argentina, a partir de diciembre 2015, con el ascenso de Mauricio Macri a la presidencia, se conformó un escenario de disputa de proyectos políticos o "hegemonía escindida" (Pucciarelli y Castellani, 2017). En ambos casos se han vaciado programas, reducido ministerios y partidas presupuestarias, siendo que en el caso de Brasil las orientaciones conservadoras se sumaron al fundamentalismo religioso y al racismo de Estado.

En lo que refiere al campo cultural, además del vaciamiento y disolución de ambos ministerios de Cultura, para el caso brasileño, esto se sumó a una política de destrucción de las manifestaciones culturales populares, construidas en el discurso público como sinónimo del "antipetismo" en su expresión más racista. En Argentina, los programas orientados hacia las culturas populares han quedado subsumidos a la Dirección de Cultura Comunitaria y sus presupuestos achicados. También se afectó la continuidad de los programas de inclusión 
socioeducativa (Centro de Actividades Infantiles, Centro de Actividades Juveniles, el mismo POyCB, entre otros), que implicó la delegación a cada jurisdicción del financiamiento argumentado en las autonomías provinciales.

En lo que respecta a las políticas de género durante el período macrista (20152019), las sinergias entre Estado y sociedad civil han estado atravesadas principalmente por la agenda de crecientes movilizaciones en temas como violencia y femicidios a partir de la campaña y movilizaciones "Ni una menos" y de las masivas manifestaciones en el año 2018 por el debate acerca de la Ley de interrupción voluntaria del embarazo, condensadas en la expresión "ola verde", en referencia al pañuelo símbolo de la Campaña por el aborto legal, seguro y gratuito. ${ }^{6}$ A fines de ese año, la denuncia de la actriz Thelma Fardin, acompañada por el Colectivo de Actrices Argentinas, trascendió el campo artístico-cultural en tanto instaló y sensibilizó a la sociedad en su conjunto acerca del acoso y abuso sexual. Cabe decir que esta puesta en agenda de temas tan caros a la reivindicación de feministas y del movimiento amplio de mujeres y disidencias no estuvo traducida en mayores presupuestos para el área, aunque se lo enuncie discursivamente.

\section{Enfoque teórico y metodológico}

Metodológicamente, siguiendo a Elena Achilli (2005), entendemos a la investigación social como un proceso dialéctico, no lineal, que supone la construcción de conocimiento científico acerca de una problemática social, mediante un enfoque que le otorga sistematicidad. En esta dirección, ambos casos analizados en el presente escrito se han construido a partir del enfoque etnográfico, priorizando la perspectiva de lxs propios actores sociales acerca de sus prácticas, representaciones y discursos. Entre las técnicas empleadas se encuentran la observación participante, la realización de entrevistas y la participación en eventos relevantes del campo.

Nos apoyamos en que las políticas públicas son construcciones sociales, históricamente situadas y que se conforman a partir de escenarios de disputa entre actores sociales con desiguales posiciones de poder en un "equilibrio de compromiso" entre unos y otros (Gramsci, 2006, p. 48). Desde esta perspectiva histórica y geopolítica, entendemos a las políticas públicas como una forma de estudiar el Estado "en acción" (Oszlak y O’Donnell, 1981, p. 104) o "en movimiento" (Raggio, 2013, p. 22), analizando qué se piensa como problema y qué no, sobre qué se interviene y sobre qué no.

Comprendida como un "campo organizacional de lo simbólico" (Ochoa Gautier, 2002, p. 5), una política cultural es una forma de intervención pública, privada y/o comunitaria en el campo cultural, cuya acción es orientada hacia una transformación o legitimación de un orden social vigente. Entendemos que dicho campo cultural se conforma a partir de la mediación entre lo político y lo social, y que no se trata exclusivamente del campo de las artes, aunque también lo comprenda. Partiendo de esta concepción preliminar, en el presente trabajo analizamos dos casos de gestión de políticas culturales referidas al lenguaje expresivo de la música, que comprenden tanto las políticas públicas culturales gestionadas desde el ámbito estatal, como aquellas políticas culturales públicas no estatales.

Es necesario resaltar de antemano que, en este amplio paraguas de las políticas culturales, no es posible equiparar el rol del Estado como garante del
6. Para una historización de la reivindicación por el derecho al aborto en Argentina recomendamos Daich y Tarducci (2018). 
derecho a la cultura con el de las organizaciones de la sociedad civil (Chauí, 2013; Raggio, 2013). Sin embargo, en el presente histórico del caso analizado en Brasil el país está atravesando un proceso de ausencia y destrucción de políticas públicas culturales y es por ello que atendemos al estudio de las políticas culturales de resistencia, desarrolladas por las organizaciones de la sociedad civil y por los sujetos allí actuantes, y cómo esta vida cultural en los "márgenes del poder" (Ortner, 2016, p. 165) expresa formas de resistencia y "poder-agencia" (Ortner, 2016, p. 167).

Siguiendo a Joan Scott (1986), entendemos al género como una categoría relacional y política, es decir, como una herramienta analítica fundamental para comprender el poder y las jerarquías sociales, que da cuenta de una de las dimensiones más invisibilizadas de la desigualdad social (Piscitelli, 1995). A la vez, la misma categoría género es desestabilizada dado que cuestiona el binarismo femenino/masculino en el marco de los estudios posestructuralistas, la teoría queer y los estudios transfeministas (Butler, 2007; Preciado, 2011; Solá y Urko, 2013).

Además, es importante señalar que los estudios de género en las políticas públicas se constituyen como un campo de investigación consolidado de larga data (Grimberg, 1999; Del Río Fortuna y Lavigne, 2009; Pozzio, 2011; Anzorena, 2013). En lo que atañe al campo de estudios de la cultura, encontramos reflexiones sobre la expresión de género en distintas manifestaciones artístico-culturales, especialmente investigaciones del ámbito de la musicología feminista, y los estudios de género sobre teoría y educación musical (Green, 2001; Ramos, 2010; Loizaga Cano, 2004). Sin embargo, en los estudios específicos de política y gestión cultural se encuentra muy poca bibliografía en relación con el género y desde una perspectiva feminista. Constituyen una excepción en este sentido los trabajos de Marcela País Andrade (2014, 2015), quien aborda "las categorías de género(s) y cultura(s) en el contexto de las Políticas de Desarrollo Cultural que las sustentan", y observa "cómo dichas categorías son (re)apropiadas desde la práctica artística, desde la Gestión Cultural y desde el campo de la investigación en cultura" (País Andrade, 2015, p. 2).

En suma, nuestra hipótesis de trabajo parte de dos postulados: entender a las políticas públicas y culturales como un campo de disputa y articulación entre la sociedad civil y el Estado, a la vez que pensar a la dimensión de género como constitutiva de las relaciones sociales. Así, la incorporación de la perspectiva de género al análisis de los procesos de gestión permite comprender cabalmente los sentidos, alcances y limitaciones de las políticas, en nuestro caso referidas a las culturales. En este sentido, sostenemos que la ausencia de una problematización respecto de las cuestiones de género en el marco de las políticas culturales tiende a reproducir desigualdades preexistentes y estereotipos hegemónicos de masculinidades, feminidades y otras identidades de género.

\section{Caso 1 - Programa de Orquestas y Coros del Bicentenario en Buenos Aires, Argentina}

Diferentes autorxs coinciden en indicar al Sistema Nacional de Orquestas Juveniles e Infantiles de Venezuela como modelo para recrear orquestas y coros juveniles en la región (Wald, 2009; Finnegan y Serulnikov, 2015). Dicho proyecto, creado en 1975 por el economista y músico José Antonio Abreu, marca un hito en las políticas sociales y culturales en Latinoamericana y también ha sido una referencia para directores y músicos a nivel global (Burgos García, 2009; Carabetta, Rincón y Serrati, P, 2017). 
En Argentina, es a fines de la década de 1990 cuando comienzan a desarrollarse orquestas pioneras en Bariloche, Chascomús y Ciudad de Buenos Aires. Vinculado estrechamente con esta última, por medio de la dirección del Maestro Claudio Espector, en 2008 se inaugura el Programa Nacional de Coros y Orquestas Infantiles y Juveniles del Bicentenario, como parte del organigrama del Ministerio de Educación de la Nación. Como tal, se extendió hasta noviembre de 2015, ya que a partir de la asunción de Mauricio Macri en diciembre de ese año, el POyCB comenzó a transitar una situación de "incertidumbre $^{\prime 7}$ fundamentada en el cambio de gobierno, pero sobre todo en el cambio de orientación del sentido de las políticas.

En este marco, el trabajo de campo realizado entre julio de 2015 y agosto de 2016 nos dio la posibilidad de participar de la transición político-institucional del programa y analizarla a partir de su impacto e implicancias en la cotidianeidad de participantes, docentes, funcionarixs y familiares. En julio de 2016, el programa se descentralizó y provincializó su organización, lógica y financiamiento. Para analizarlo en su dinámica más propia de funcionamiento, ${ }^{8}$ tomaremos fragmentos de campo del periodo julio-diciembre de 2015.

En su definición, el POyCB se planteaba como objetivo la "inclusión social" a partir de la articulación entre lo educativo, lo social y lo cultural. Entre los años 2008 y 2015, se crearon más de 150 orquestas y 150 coros, en 22 de las 24 jurisdicciones provinciales. ${ }^{9}$ Los espacios físicos para realizar las actividades fueron en su mayoría escuelas primarias o secundarias de gestión estatal. La convocatoria estaba orientada a niñxs y jóvenes en edad escolar, tanto de nivel primario como secundario, estudiaran o no en la escuela donde funcionaba la orquesta y estuvieran o no escolarizados. La conformación del plantel docente incluía un director/a, docentes de lenguaje musical, percusión, cello, violín/ viola, contrabajo, flauta traversa, clarinete, oboe, trompeta, trombón, y un/a docente integrador/a con un perfil de articulación sociocomunitario.

Entre los aspectos más valorados por lxs participantes se destacaron las clases con profesorxs idóneos y reconocidos, el préstamo o comodato de los instrumentos, así como la participación en eventos, viajes y capacitaciones.

La orquesta tomada como caso se creó en 2012 en un barrio del conurbano bonaerense con baja oferta de propuestas culturales desde el Estado. En el transcurso del período de observaciones participaban alrededor de 40 chicxs de entre 6 y 17 años en las actividades regulares.

\section{Caso 2 - Las rodas de samba en Río de Janeiro, Brasil}

El samba es una manifestación cultural de matriz afro cuyo origen remonta a las primeras décadas del siglo XX en la zona portuaria de Río de Janeiro y, según cuentan nuestros interlocutores, se ha ido conformando a partir del encuentro entre exesclavos que migraban hacia la entonces capital del país en búsqueda de trabajo y las particularidades de la vida urbana. De acuerdo con Marcos André, gestor cultural brasileño y uno de nuestros interlocutores en el trabajo de campo, en las calles de Río de Janeiro de aquel entonces confluyeron distintas manifestaciones populares de otras regiones de Brasil, tales como el jongo, el candomblé, la umbanda y el afoxé, y es a partir de ese encuentro que aparecen las rodas de samba en las calles de la ciudad. Según el documento de declaratoria del samba como Patrimonio Cultural de Brasil (IPHAN, 2015), la tradición de las danzas en ronda tiene su origen en los pueblos bantús del
7. "Orquestas en plan de lucha. Futuro incierto". https://www.pagina12.com.ar/9932-futuro-incierto (20/11/19)

8. En noviembre de 2016, la denominación del Programa pasa a ser Programa Nacional de Coros y Orquestas Infantiles y Juveniles "Música para la Equidad" y se designa como coordinador al licenciado Eduardo Andrés Ihidoype. Decisión administrativa 1236/2016 Jefatura de Gabinete de Ministros. Disponible en: http://servicios. infoleg.gob.ar/infoleglnternet/verNorma.do?id=267135 (29/11/19)

9. Exceptuando la provincia de Misiones y la Ciudad de Buenos Aires, según un relevamiento propio. 
10. Decreto Municipal $n^{\circ} 41.036$. 01 de diciembre de 2015. Río de Janeiro, Brasil. antiguo Congo que han sido sometidos a la migración forzada y al despojo en la trata atlántica entre los siglos XV y XIX.

En la actualidad, por más que el samba tenga distintas vertientes y se haya desarrollado en todo Brasil, el formato roda de samba es una manifestación cultural particular de Río de Janeiro, casi como un sello identitário de la ciudad. Si bien cada roda tiene su particularidad según el barrio/región en que sucede y de acuerdo con quienes la organizan, en general, acontecen en una plaza, en una calle, delante o dentro de un bar, en el patio de una Escola de samba ("terreiro"), en un centro cultural; entre otras múltiples y diversas posibilidades de espacios para su realización. La primera roda en que participamos tenía lugar en una plaza, al lado de una tradicional feria de frutas y verduras en el barrio de Glória, zona sur de Río de Janeiro. Bajo una lona -armada en caso de lluvia-, se agrupaban algunas mesas al centro del espacio, alrededor de las cuales se ubicaban los músicos sentados con sus instrumentos: guitarra, cavaquinho, banjo y diversos instrumentos de percusión, como pandeiro, surdo, cuíca, tamborín, chocalho, reco-reco, repique, tam-tam, entre otros. Al lado, una mesa de sonido para los micrófonos y parlantes. Alrededor, mesas organizadas en círculos para el público asistente completando toda la circunferencia de la lona, y luego, algunos puestos de comidas típicas y bebidas. Un segundo círculo imaginario estaba conformado por personas paradas cantando, bailando y circulando por la plaza.

Por lo general, estas rodas de samba no cuentan con un financiamiento estatal directo, sino que se trata de formas de producción cultural promovidas y autogestionadas por la comunidad, que se apoyan en la venta de comidas y bebidas para su mantenimiento. Esta situación de cierta vulnerabilidad y ausencia de políticas públicas culturales expone esta manifestación cultural popular a acciones represivas, que muchas veces dependen de la buena relación entablada con la policía o los agentes estatales de control del espacio público. Frente a esta situación de desprotección, diversos colectivos de rodas de samba se agruparon para conformar la Rede Carioca de Rodas de Samba a fines del año 2014, y lograron, por medio de un Decreto municipal del año $2015,{ }^{10}$ gestionar un subsidio para 450 rodas en toda la ciudad. Sin embargo, esto aún es una acción muy puntual que, tal como nos contó una de sus impulsoras, todavía depende de la buena voluntad del gobierno de turno y no se trata de una política cultural pública sostenida.

\section{Estereotipos y desigualdades de género en la música y en su gestión}

A continuación, nos proponemos abordar las experiencias en tanto identidades, estereotipos, roles, prácticas y discursos de género en el lenguaje musical y en su gestión político-cultural.

Lucy Green, socióloga británica, pionera en el campo de la educación musical, analizó el género en relación con la producción y recepción contextualizada de la música y sostiene que "en virtud de cómo es usada en la sociedad, la música puede 'significar' (o como yo lo he llamado: 'delinear' [delineate]) género -es decir: masculinidad, femineidad y todos sus complejos matices" (Carabetta, 2016, Entrevista con Lucy Green, p. 137).

Por su parte, Alejandra Quintana (2006), investigadora colombiana, lo expresa de la siguiente forma: 
La música ha personificado a lo largo de la historia cambios y permanencias en las construcciones de género; a través de ella, se han pronunciado aquellas actitudes que han permanecido relativamente constantes frente al ser hombre o al ser mujer; lo masculino fuerte, objetivo y racional, frente a lo femenino débil, subjetivo y corporal, bipolaridades supuestas que, así no sean material propio de los sexos, son impuestas social y culturalmente y se perpetúan, también, a través de las manifestaciones artísticas. (p. 4)

En el caso del $\mathrm{POyCB}$, destacamos en primer lugar que los documentos oficiales no se refieren explícitamente a cuestiones de género, sino que los principales objetivos están vinculados a la tríada de políticas: social, educativa y cultural. En referencia a estos, se mencionan el acceso a la música como bien cultural, así como específicamente la retención y reinserción en el plano educativo. Sintetizados en el término "inclusión", el aspecto social expresa y condensa estos significados a la vez que advierte desigualdades socioeconómicas.

En el transcurso del trabajo de campo indagamos sobre la percepción que lxs diversos actores tiene acerca de la igualdad de género en el espacio de la orquesta. En líneas generales, el espacio se representa como igualitario en lo que refiere a la participación de "chicas y chicos". Aunque fueron los docentes integradores quienes problematizaron algunas prácticas, como la elección de los instrumentos:

En las orquestas hay algunos instrumentos que están como feminizados y asociados a lo débil, entonces ubica a las mujeres [niñas] en ese lugar y los niños/jóvenes/ varones se ubican en instrumentos que están más asociados a lo masculino. Entonces tenés un montón de chicas tocando el violín porque suena fino, como una cosita que se va a romper, y tenés a los varones en las trompetas, la percusión, el contrabajo, que son instrumentos grandes. (Entrevista a Mariano, agosto/2015)

En concordancia con lo anterior, la observación etnográfica permitió relevar situaciones como "cargadas" y burlas entre participantes que dan cuenta del atravesamiento del espacio de la orquesta por los sentidos hegemónicos respecto de la heteronormatividad y estereotipos de género. En tono risueño y acusatorio, un participante le decía a otro: “Vos no tocas el violín, sos violín"11 (Entrevista a Lucas, octubre/2015). Mientras, una participante niña lo expresaba de este modo: "A una nena que toca la trompeta otra nena la cargaba, le decía: Vos sos remarimacho, tocás la trompeta. Y a un nene que toca el violín le dicen P.U.T.O (deletrea) porque los chicos piensan que es gay" (Entrevista a Brenda, octubre/2015).

Así, tanto en la elección de los instrumentos como en las representaciones y discursos de lxs participantes hallamos nudos significativos donde se expresan las tensiones de una política de "inclusión social" que está atravesada por la dimensión de género. En este sentido, advertimos que los procesos de gestión no son genéricamente neutros.

En el caso de análisis del samba en Río de Janeiro, ante la pregunta de cómo es ser mujer y trabajar con samba, una cantante nos contesta en primer lugar que: "El samba es el reducto cómodo del machismo. Y dentro de todas las manifestaciones culturales y géneros musicales es el más cómodo [...]. Para romper con eso, tienes que hacer el doble para ser reconocida por la mitad" (Entrevista a Marina, julio/2019).
11. Violín es un término peyorativo muy difundido en el lenguaje popular que proviene del lunfardo carcelario y significa violador. 
12. La expresión café con leche es utilizada en distintos juegos de niñxs para referirse a aquel niñx que aún no tiene la edad suficiente para participar del juego oficialmente, entonces los de más edad le permiten que entre en el juego, pero su participación no es considerada competitiva.
Ese machismo es vivenciado por ella según una relación de tutelaje del hombre músico por sobre la mujer música: "los tipos te abrazan, te traen para dentro de sus espacios de una forma realmente tutelar" (Entrevista a Marina, julio/2019). Cuenta que muchas veces, cuando invitan a una mujer a la roda de samba ya le dicen qué canción debe cantar, presuponiendo que ella, como mujer, no tendrá la capacidad de elegir.

Las situaciones de tutelaje o de considerar la mujer como un sujeto incompleto, casi como un niñx, se multiplican. Marina nos cuenta una situación en la que a ella, mientras sostenía un vaso de cerveza en la mano mientras cantaba, un músico varón allí presente le dijo: "dame el vaso", a lo que ella al micrófono contestó, apuntándole: "Acá está mi nutricionista!" y siguió cantando, valiéndose de la burla como una forma de resistencia.

Un tercer nivel de desigualdad de género señalado por la cantante sucede cuando se les exige a las mujeres que, si van a tocar un instrumento, sean muy buenas haciéndolo, demanda que no se les hace a los varones: "Es obvio que no todas las mujeres van a ser las exponentes de su instrumento. Vas a una roda de varones y nadie está preocupado si fulano es el mejor del mundo en su instrumento" (Entrevista a Marina, julio/2019).

Lo mismo es señalado por una productora cultural de samba:

\begin{abstract}
La mujer es invisibilizada. La mujer es café con leche. ${ }^{12}$ Vos llegás a una roda, llega un tipo para tocar y toca peor que vos y todos dicen: "que copado, el tipo está aprendiendo". Llega una mujer y toca mal en la roda y dicen: "iQue mal que toca esa mina, horrible!". Es muy loco ver que la mujer tiene que ser tres veces mejor que el hombre para poder ser ok. (Entrevista a Patricia, julio/2019)
\end{abstract}

Es cierto que esto se relaciona con una desvalorización del trabajo de las mujeres en el samba y en la producción cultural en general. Asimismo, en los últimos años, junto con la transversalización de las reivindicaciones de género se vienen realizando diversas rodas de samba producidas y gestionadas por mujeres; entre ellas nombramos: É samba que elas querem e Moça Prosa. Otra iniciativa de resistencia ha sido la implementación del Dia da Mulher Sambista (Ley 6454/2019), una iniciativa del Movimento das Mulheres Sambistas, impulsada por el legislador Tarcísio Motta, que instaura el día 13 de abril -natalicio de la compositora Dona Ivone Lara- como el día de celebración a la mujer sambista en la ciudad de Río de Janeiro. Con respecto a esta política cultural, una de las participantes del movimiento cuenta que es una iniciativa para que las mujeres se organicen y luchen por sus derechos en el samba, ya que "el movimiento convoca diciendo: Vení mujer, acá hay un lugar para vos" (Entrevista a Patricia, julio/2019).

A partir de esta ley, la colectiva de mujeres logró la aprobación de dos proyectos de fomento a la cultura de la municipalidad de Río de Janeiro y un financiamiento de una institución alemana. Conforme lo cuenta Patricia, este último se logró en gran parte debido a que la gestora de dicha ONG también era una mujer y "se sintió identificada con el proyecto" (julio/2019).

Asimismo, en lo que atañe a este movimiento contracultural vivo y potente, Patricia nos cuenta que:

Vas a las rodas de samba de mujeres acá en Río y no ves artistas, músicos o cantores varones. Pero cuando vas a una roda de samba de varones, todos los tipos que no 
estén trabajando ese día están ahí prestigiando sus colegas. Pero nosotras hicimos el Dia da Mulher Sambista, convocamos a más de 5 mil personas en Cinelândia ${ }^{13}$ y que recuerde vi un solo cantor famoso. En noviembre tuvimos en Encontro Nacional das Mulheres Sambistas y tampoco había músicos varones en el público. (julio/2019)

En suma, a partir de los dos casos descritos hemos podido observar cómo la ausencia de una problematización de género en la gestión y en el quehacer cultural conlleva a la reproducción de desigualdades y estereotipos hegemónicos de masculinidades y feminidades. Sin embargo, esto no inhabilita el surgimiento y la expresión de prácticas que impugnan, cuestionan y desarrollan estrategias de resistencia y posible transformación social.

\section{Maternidad/trabajo cultural - ¿mujer-madre?}

A continuación, atendemos a la aparición del estereotipo de género construido con respecto a las mujeres a partir de los binomios maternidad/cuidados y maternidad/trabajo. Con respecto a esto, sostenemos junto con Marcela Nari que:

La maternidad incluye una serie de procesos biológicos (concepción, embarazo, parto, puerperio y, en algunos casos, lactancia) pero se extiende mucho más allá de ellos, hacia prácticas y relaciones sociales no vinculadas al cuerpo femenino (cuidado y socialización, atención de la salud, alimentación, higiene, afecto y cariño). Todas estas prácticas se encuentran entrelazadas con representaciones acerca de lo socialmente aceptado, legitimado, "naturalizado". (Nari, 2004, p. 17)

En primer lugar, el binomio maternidad/cuidados refiere a la traspolación de las prácticas y relaciones sociales vinculadas a la posibilidad biológica de la concepción y sus consecuentes procesos, hacia prácticas y relaciones sociales en las cuales se naturaliza el rol de mujeres como madres en todos los aspectos de la vida social: maternar espacios, relaciones de cuidado, entre otros.

En el caso del samba, cuando le preguntamos a una productora cultural sobre cómo es el rol de la mujer en el samba hoy, nos contestó también que hay una dificultad al interior de ese movimiento cultural en ver a la mujer como "dueña del pagode", como se dice en Brasil, lo que significa una mujer que ocupa un rol de poder, que en una sociedad patriarcal está asociado a la masculinidad y al accionar en el espacio de lo público. Nos cuenta que, si una mujer ocupa un espacio de poder en la gestión y en la política cultural, lxs demás involucrados la etiquetan como "lesbiana". Esta etiqueta está basada en una idea/prejuicio social que opera en este caso como una acción del grupo sobre la persona (Becker, 2009), a partir de una transpolación de características socialmente valoradas y asignadas al "hombre proveedor" hacia una mujer con una orientación sexual que socialmente aparece asociada a "lo masculino". De acuerdo con Marta Lamas (1986) la división sexual del trabajo basada en la oposición del ámbito privado-femenino y ámbito privado-masculino construye roles, normas y prescripciones rígidas que "condicionan los papeles y limitan las potencialidades humanas de las personas" (p. 7).

Relacionado con esta división prescripta socialmente, Patricia también relata que en el mundo del samba hay pocas mujeres en el rol de producción, y las que están son, por lo general, productoras artísticas; muy pocas son productoras ejecutivas. Según su experiencia como mujer en un espacio de coordinación y producción ejecutiva de proyectos culturales, cuenta que muchas veces la 
etiquetan como totoca - palabra que en portugués significa "loca" - cuando impone su voluntad en un trabajo y exige que la paguen lo que corresponde.

La misma productora cultural nos cuenta que, debido a esta situación, en distintos espacios del samba, la mujer se encuentra subsumida al rol de madre, de cuidados, "de la tía de la comida", "la tía de la limpieza", de garantizar que las cosas funcionen bien, pero sin tomar protagonismo. En esta dirección, en una de las reuniones del Movimento das Mulheres Sambistas, un músico famoso tomó la palabra y dijo: "Me parece muy lindo este movimiento, hasta me gusta la mujer en la producción cultural, porque la mujer tiene esa mirada de ver una basurita en el piso y levantarla" (julio/2019).

En el caso del Programa de Orquestas y Coros, su gestión no contaba con personal de limpieza ni cocina ("auxiliares", según la definición de la Dirección General de Escuelas de la Provincia de Buenos Aires). Estas tareas eran realizadas por grupos ad hoc de "mamás colaboradoras", según el registro discursivo de docentes y funcionarixs del programa. En el caso de la orquesta analizada, ellas se autodenominaban "matecocideras" (Entrevista a Laura, noviembre/2015). Las tareas principales de este equipo de mujeres eran preparar las meriendas los días de ensayo, acompañar la entrada y salida de lxs participantes en la escuela, los viajes y las presentaciones. Realizaban también tareas de limpieza y el orden de los espacios utilizados después de los ensayos, en conjunto con docentes y participantes. Uno de los docentes indicaba diferentes motivos por los cuales estas "mamás" comenzaron a participar: curiosidad, cuidado, ganas de compartir. Enfatizando las diferencias en la participación de "mamás" y "papás", agregaba:

Digo grupo de mamás, porque son concretamente mamás, no porque son mamás y haya papás... son mamás y están ahí y hacen la merienda y cuando hacemos algún evento nos acompañan, llevan los instrumentos y toman la posta de ver que estén todos, de ayudarnos que los pibes no se olviden nada. Ese espacio se dio adentro de la orquesta, autogestionado digamos... (Entrevista a Mariano, agosto/2015)

Consultadas "las mamás" acerca de la participación de adultxs varones/padres en el espacio de colaboración, descartaban esta posibilidad, indicando desinterés. Así, la subsunción de estas "mujeres" en "madres", tanto como la adjetivación de "colaboradoras", encajan en las mencionadas ideas y valores relacionados con la maternidad como práctica de cuidado y socialización que se extiende más allá de la familia, expresadas aquí como trabajo solidario y gratuito.

Un segundo grupo de sentido construido a partir del binomio maternidad/ trabajo refiere tanto a las discriminaciones y valoraciones negativas ejercidas por sobre las mujeres como madres negligentes a la hora de ejercer un trabajo cultural, así como a la precarización laboral al momento de la maternidad y sus consecuentes procesos.

También Patricia, la productora cultural brasileña, cuenta que cuando está trabajando a la noche todos preguntan dónde está su hija y duda si la misma pregunta es realizada a los varones que son padres. A su vez, la cantante Marina nos cuenta que:

No tengo ningún amigo hombre que haya dejado de tocar por tres meses debido al nacimiento de un hijo. Tenés que entender que la mujer tiene que parar de tocar en ese periodo (de gestación, parto y lactancia), pero que además cuando vuelve a la 
roda va ser juzgada por todo el mundo, que le preguntan: “¿dónde está el bebé?”. Pero cuando llevan el bebé al evento, empiezan a decir: “cuidado con el oído...”. (julio/2019)

En referencia al POyCB señalamos una tensión entre el derecho a la cultura (acceso al bien cultural) y el derecho de la cultura (de lxs trabajadores culturales). Así, mientras que el programa garantiza los primeros, vulnera los segundos mediante la contratación precaria de sus trabajadorxs. En este sentido, la situación de las docentes mujeres que deciden ser madres es de mayor vulnerabilidad que la de sus compañeros varones. Graficando esta desventaja, una de las integrantes del equipo fundacional de la orquesta, renunció estando embarazada de siete meses:

Me voy a quedar trabajando en un colegio que estoy hace muchos años, en blanco y me pagan toda la licencia, acá no me conviene. Igual hablé con el director y quedamos que capaz pueda volver al año que viene. El problema es muy flexible, hay mucho recambio, si se genera un lugar el año que viene capaz vuelvo. (Entrevista a Josefina, agosto/2015)

Así, si bien la precarización laboral fue tematizada como malestar y/o demanda, la vulneración de las trabajadoras mujeres estuvo naturalizada e invisibilizada: no figuró como reclamo específico de sus protagonistas, ni tampoco del Colectivo de Trabajadores de Orquestas y Coros del Bicentenario. ${ }^{14}$ Para finalizar, y poniendo en perspectiva la cita, cabe reiterar que a partir de 2016, la asunción del Frente Cambiemos redefinió un nuevo modelo de acumulación y de "proyecto hegemónico conservador-neoliberal" (Pucciarelli y Castellani, 2017, p. 16), que reconfiguró el campo de las políticas públicas en todos los ámbitos del Estado, así como su entramado con la sociedad civil.

\section{Conclusiones}

En este trabajo hemos realizado un análisis inicial de cómo se expresa la cuestión de género en el quehacer cotidiano y en la gestión de políticas de la cultura orientadas al lenguaje expresivo de la música, a partir de dos casos: el Programa de Orquestas y Coros Infantiles y Juveniles del Bicentenario (POyCB) - Argentina - y el samba en tanto movimiento cultural contemporáneo de artistas, trabajadorxs de la cultura y gestorxs culturales en la ciudad de Río de Janeiro - Brasil.

A partir de la descripción y análisis de datos construidos en trabajo de campo en los dos casos, hemos atendido a cómo la ausencia de una problematización de género en la gestión y en el quehacer cultural conlleva a la reproducción de desigualdades y estereotipos hegemónicos de masculinidades, tales como lo masculino asociado a lo "fuerte", "objetivo", "racional", frente a lo femenino asociado a lo "débil", "subjetivo" y como un objeto de tutelaje; que conlleva a un esquema bipolar de los roles de género.

Atendimos a cómo todavía se sigue reproduciendo esta división ancestral del ámbito privado-femenino y ámbito público-masculino, que orienta roles de género construidos a partir de normas y prescripciones heteronormativas, por ejemplo, la imposibilidad de la mujer de ser "dueña del pagode" y la cristalización en los roles de "tía de la limpieza" y "tía de la comida" en el samba de Río de Janeiro. Asimismo, también hemos constatado que esto no inhabilita la emergencia de prácticas que impugnan, cuestionan y desarrollan estrategias
14. Este colectivo se creó en 2012 y por la trascendencia que adquirió en la segunda etapa del trabajo de campo (enero-agosto 2016), producto de la asunción de Mauricio Macri en diciembre 2015, fue un actor central en este último periodo. 
de resistencia y posible transformación social, tales como las rodas de samba de mujeres y el Movimento das Mulheres Sambistas.

En segundo lugar, dimos cuenta de la aparición del estereotipo de género construido con respecto a las mujeres a partir del binomio maternidad/cuidados. Retomando a Marcela Nari (2004), observamos cómo la maternidad se extiende más allá de los procesos biológicos a otras prácticas y roles sociales, que naturalizan el rol de la mujer como madre en varios ámbitos de la vida social, sean estos trabajos remunerados o no. En el caso del POyCB observamos cómo el Estado no es neutro, sino que se beneficia del trabajo comunitario, gratuito y doméstico (Anzorena, 2013) de las "mamás colaboradoras". Remarcamos que esto no supone una falta de agencia por parte de ellas, aunque esta línea de análisis no haya sido abordada en el presente escrito.

Luego, hemos señalado cómo las mujeres trabajadoras culturales son consideradas como negligentes o malas madres, por ejemplo, a partir de la pregunta “¿dónde está el bebé?”. Además, documentamos la precarización laboral de las trabajadoras culturales, como en el caso del POyCB.

En esta dirección, podemos concluir que la ausencia de problematización en términos de género reproduce las desigualdades preexistentes, ya que impide democratizar las barreras simbólicas que constriñen los roles tradicionales en esta materia. Por lo tanto, pensando a la dimensión de género como constitutiva de las relaciones sociales, postulamos que la incorporación de la perspectiva de género al análisis de los procesos de gestión permitió comprender los sentidos, alcances y limitaciones de dichas políticas y movimientos culturales.

Para finalizar, remarcamos los aportes de la crítica feminista situada y de la perspectiva analítica de los estudios de género a las políticas culturales en clave antropológica. Asimismo, advertimos que no es posible desvincular las cuestiones de género de tramas interseccionales, en un análisis transversal que considere además la clase social y la pertenencia étnica. En este sentido, compartimos la afirmación de Donna Haraway (2018): “La conciencia de género, raza o clase es un logro forzado en nosotras [mujeres, feministas] por la terrible experiencia histórica de las realidades sociales contradictorias del patriarcado, del colonialismo y del capitalismo." (p. 23)

A modo de cierre, esperamos contribuir con este escrito a la ampliación de las demandas por derechos culturales, sociales y económicos, así como por su transversalización en términos de género.

\section{Financiamiento:}

Este documento es resultado del financiamiento otorgado por el Estado Nacional, por lo tanto, queda sujeto al cumplimiento de la Ley № 26.899.

Organismo Financiador: Universidad de Buenos Aires. Programa UBACyT Programación 2018-2021. Código del Proyecto: 20020170100025BA. Nombre del Proyecto: "La continuidad de las políticas públicas designadas en términos de inclusión y participación, rupturas y reelaboraciones en el nuevo contexto político nacional. Aportes desde una mirada antropológica." 
Organismo Financiador: Universidad de Buenos Aires. Beca UBACyT categoría doctorado 2018-2021. Código de proyecto: 20220180100063BA. Nombre del Proyecto: "Cultura, desarrollo e identidad: políticas culturales en Buenos Aires y Río de Janeiro desde 1960 hasta la actualidad".

\section{Agradecimientos:}

Agradecemos a la Universidad de Buenos Aires y al Proyecto UBACyT "La continuidad de las políticas públicas designadas en términos de inclusión y participación, rupturas y reelaboraciones en el nuevo contexto político nacional. Aportes desde una mirada antropológica".

\section{Sobre las autoras}

Diana Bento de Mello es licenciada en Ciencias Antropológicas (UBA). Becaria doctoral (2018-2021) del Instituto de Ciencias Antropológicas, Facultad de Filosofía y Letras (UBA) en el marco de la investigación UBACyT "La continuidad de las políticas públicas designadas en términos de inclusión y participación, rupturas y reelaboraciones en el nuevo contexto político nacional. Aportes desde una mirada antropológica", que integra el Programa Economía Política de la Cultura. Estudios sobre Producciones Culturales y Patrimonio de la misma Universidad.

Alejandra Vázquez es profesora y licenciada en Ciencias Antropológicas (UBA). Integra el UBACyT "La continuidad de las políticas públicas designadas en términos de inclusión y participación, rupturas y reelaboraciones en el nuevo contexto político nacional. Aportes desde una mirada antropológica", radicado en el Programa Economía Política de la Cultura. Estudios sobre Producciones Culturales y Patrimonio de la misma Universidad. 


\section{Q Referencias bibliográficas}

»Achilli, E. (2005). Investigar en Antropología Social. Los desafíos de transmitir un oficio. Rosario: Laborde.

»Anzorena, C. (2013). Mujeres en la trama del Estado. Una lectura feminista de las políticas públicas. Mendoza: EDIUNC.

» Becker, H. (2009). Outsiders. Buenos Aires: Siglo XXI.

» Becker, H. (2016). Mozart, el asesinato y los límites del sentido común: Cómo construir teoría a partir de casos. Buenos Aires: Siglo XXI.

» Burgos García, O. (2009). El eco de la orquesta. Análisis de los valores humanos reflejados en las crónicas sobre las presentaciones de la Orquesta Sinfónica de la Juventud Venezolana Simón Bolívar. Málaga: Universidad de Málaga.

» Butler (2007). El género en disputa. El feminismo y la subversión de la identidad. Barcelona: Paidós.

" Carabetta, S. (2016). Entrevista con Lucy Green. Foro de educación musical, artes y pedagogía, Vol. 1 (Núm. 1), pp. 133-156.

»Carabetta, S., Rincón, C., y Serrati, P. (2017). Entrevista con J. Abreu y E. Méndez, de 'El Sistema' de Orquestas (Venezuela). Foro de educación musical, artes y pedagogía, 2(2), 131-146.

»Chauí, M. (2013). Ciudadanía cultural. El derecho a la cultura. Buenos Aires: RGC libros.

» CGLU (2018). La cultura en los objetivos de desarrollo sostenible: guía práctica para la acción local. Recuperado de www.agenda21culture.net/

»Daich, D. y Tarducci. M. (2018). Antropólogas feministas por el derecho a decidir. Aportes para una historia de la lucha por la despenalización y legalización del aborto en Argentina. Publicar, 16(24) Recuperado de ppct.caicyt.gov.ar/index.php/publicar/article/ viewFile/14144/45454575758950

» Del Río Fortuna, C. y Lavigne, L. (2009). Una mirada antropológica de dos políticas públicas en sexualidad en la Ciudad de Buenos Aires. Propuesta Educativa, 33, 95-104.

" Finnegan, F. y Serulnikov A. (2015). La enseñanza y el aprendizaje como ejes de la propuesta socioeducativa. El Programa Nacional de Orquestas y Coros Infantiles y Juveniles para el Bicentenario. Dirección Nacional de Información y Evaluación de la Calidad Educativa. Serie La Educación en Debate 20.CABA

» García Canclini, N. (1987). Políticas culturales en América Latina. México: Grijalbo.

»Gramsci, A. (2006). Los intelectuales y la organización de la cultura. Recopilación Iván Valdez Jiménez. Recuperado de: www.gramsci.org.ar

» Green, L. (2001). Música, género y educación. Trad. Pablo Manzano. Madrid: Morata.

» Grimberg, M. (1999). Sexualidad y relaciones de género: una aproximación a la problemática de la prevención al Vih-Sida en sectores populares de la ciudad de Buenos Aires. Cuadernos Médico Sociales, 75, 65-76.

" Haraway, D. (2018). Manifiesto para cyborgs. Ciencia, tecnología y feminismo socialista a finales del siglo XX. Mar del Plata: Letra Sudaka.

"IPHAN. (2015). Dossier das Matrizes do Samba no Rio de Janeiro. Recuperado de portal. iphan.gov.br/ 
" Lamas, M. (1986). La antropología feminista y la categoría de género. Nueva Antropología, 8(30), 173-198.

" Loizaga Cano, M. (2004). Los estudios de género en la educación musical. Revisión crítica. Musiker, 14, 159-172.

» Menicucci de Oliveira, E. (2013). Dez anos de política para as mulheres: avanços e desafios. En E. Sader (org.), 10 anos de governos pós-neoliberais no Brasil: Lula e Dilma (pp. 323-336). San Pablo: Boitempo.

» Nari, M. (2004). Políticas de maternidad y maternalismo político. Buenos Aires 1890-1940. Buenos Aires: Biblos.

" Nivón Bolán, E. (2013). Las políticas culturales en América latina en el contexto de la diversidad. En: A. Grimson y K. Bidaseca, K (coord.). Hegemonía cultural y políticas de la diferencia (pp. 23-45). Buenos Aires: Consejo Latinoamericano de Ciencias Sociales.

"Ochoa Gautier, A. M. (2002). Políticas culturales, academia y sociedad. En D. Mato (coord.), Estudios y Otras Prácticas Intelectuales Latinoamericanas en Cultura y Poder (pp. 213-224). Caracas: Consejo Latinoamericano de Ciencias Sociales.

" Ortner, S. (2016). Antropología y teoría social: cultura, poder y agencia. San Martín: Universidad Nacional de General San Martín.

» Oszlak, O. y O’Donnell, G. (1981). Estado y políticas estatales en América Latina: hacia una estrategia de investigación. Redes. Revista de estudios sociales de la ciencia, 2(4), 98-128.

"País Andrade, M. (2014). "Lo cultural” desde una perspectiva de género. Políticas, Desarrollo y Diversidad. Horizontes Sociológicos. Revista de la Asociación Argentina de Sociología, 4(4), 125-137.

"País Andrade, M. (2015). Avances y limitaciones en la política cultural argentina y su gestión desde una perspectiva de género. En Pensar la Cultura Pública. Apuntes para una cartografía nacional (pp. 18-35). Buenos Aires: Ministerio de Cultura de la Nación.

"Piscitelli, A. (1995). Ambigüedades y desacuerdos: los conceptos de sexo y género en la antropología feminista. Cuadernos del Instituto Nacional de Antropología y Pensamiento Latinoamericano, 16, 153-169.

"Pozzio, M. (2011). Madres, mujeres y amantes. Usos y sentidos del género en la gestión cotidiana de las políticas de salud. Buenos Aires: Antropofagia.

"Preciado, B. (2011). Manifiesto contrasexual. Barcelona: Anagrama

» Pucciarelli, A. y Castellani, A. (2017). Introducción. El kirchnerismo y la conformación de un régimen de hegemonía escindida. En A. Pucciarelli y A. Castellani (comps.), Los años del kirchnerismo. La disputa hegemónica tras la crisis del orden neoliberal. Buenos Aires: Siglo XXI editores, 16-31.

》Quintana, A. (2006). Género, poder y tradición. Al baile de la gaita el tambor le replica (Tesis de Maestría). Facultad de Ciencias humanas, Universidad Nacional de Colombia, Colombia.

" Raggio, L. (2013). Las relaciones entre el campo cultural y el campo del poder. Las políticas culturales en la Ciudad de Buenos Aires 2000-2010 (Tesis doctoral). Facultad de Filosofia y Letras. Universidad de Buenos Aires, Buenos Aires, Argentina.

"Ramos, P. (2010). Luces y sombras en los estudios sobre las mujeres y la música. Revista Musical Chilena, LXIV(213), 7-25.

"Scott, J. (1996). El género: una categoría útil para el análisis histórico. En M. Lamas (comp.), El género. La construcción cultural de la diferencia sexual. México: UNAM. 265302. 
» Solá, M. y Urko, E. (comps.). (2013). Transfeminismos. Epistemes, fricciones y flujos. Txalaparta: Tafalla.

» Tarducci, M. y Rifkin, D. (2010). Fragmentos de historia del feminismo en Argentina. En Chaher, S. y Santoro, S. (comps.). Las palabras tienen sexo II. Buenos Aires: Artemisa Comunicación, 17-39.Recuperado de http://escuelasociopoliticadegenero.blogspot. com/2014/o7/fragmentos-de-historia-del-feminismo-en.html

»Wald. G. (2009). La cultura como recurso. Sentidos y apropiaciones de dos programas de orquestas juveniles de la Ciudad de Buenos Aires (Tesis doctoral). Facultad de Ciencias Sociales, Universidad de Buenos Aires, Buenos Aires, Argentina.

»Wittig (2006 [1992]). El pensamiento heterosexual y otros ensayos. Madrid: EGALES. 